\title{
A Call for Reform: Variability and Insufficiency in Radiation Oncology Resident Didactics—a Brief Report and National Survey of Program Directors
}

\author{
Matthew J. Abrams ${ }^{1,2}$ (D) Daniel W. Golden ${ }^{3} \cdot$ Grace C. Huang ${ }^{2,4}$
}

Accepted: 8 August 2021 / Published online: 18 August 2021

(c) American Association for Cancer Education 2021

\begin{abstract}
An informal needs assessment and lack of a national standardized curriculum suggest that there is tremendous variability in the formal teaching of radiation oncology resident throughout the USA. The goal of this study was to characterize formal radiation oncology resident education, in order to identify knowledge gaps and areas for improvement. We developed a 14-item survey consisting of the following domains: program characteristics, teaching faculty, formal teaching time, instructional approaches for formal teaching, curricular topics, and satisfaction with didactics. All 91 accredited US-based radiation oncology program directors received an invitation to complete the survey anonymously by email. Twenty-four (26\% response rate) program directors responded. Programs used a variety of instructional methods; all programs reported using lecturebased teaching and only a minority using simulation (38\%) or flipped classroom techniques (17\%). Other than PowerPoint, the most common electronic resource utilized was quizzing/polling (67\%), webinar (33\%), and econtour.org (13\%). The lack of a national, standardized, radiation oncology residency didactic curriculum promotes variability and insufficiency in resident training. Themes for improvement were diversity in didactic topics, incorporation of evidence-based teaching practices, increased faculty involvement, and sharing of resources across programs. Development of a national curriculum and increased electronic resource sharing may help address some of these areas of improvement.
\end{abstract}

Keywords Radiation oncology $\cdot$ Residents $\cdot$ Graduate medical education $\cdot$ Survey $\cdot$ Program directors

\section{Introduction}

Radiation oncology residency training consists of an internship year (usually internal medicine, surgery, or transitional year internship), followed by 4 years of dedicated radiation oncology training. As with other specialties, formal resident didactics are an essential part of the training process, in addition to hands-on experience. Residents take board

Matthew J. Abrams

Mabrams2@bidmc.harvard.edu

1 Department of Radiation Oncology, Beth Israel Deaconess Medical Center, 330 Brookline Avenue, Boston, MA 02215, USA

2 Harvard Medical School, Boston, MA, USA

3 Department of Radiation and Cellular Oncology, The University of Chicago, Chicago, IL, USA

4 Department of Medicine, Beth Israel Deaconess Medical Center, Boston, MA, USA examinations in radiation biology, radiation physics, and clinical radiation oncology. While national didactic curricula and topical outlines from the American Society of Radiation Oncology (ASTRO) are available for radiation biology and physics, only single institutions have published their experience with a curriculum for clinical radiation oncology [1-3].

An informal needs assessment conducted with radiation oncologists (phone and in-person) has suggested that there is tremendous variability and insufficiency in clinical radiation oncology resident didactics throughout the USA. Recently, there has been an increase in published manuscripts detailing the inadequacies in current radiation oncology residency curricula, which often omit topics of contemporary relevance, such as financial health, burnout, genomics bioinformatics and immunology, palliative care, gross anatomy, and quality improvement [4-8]. Additionally, these curricular guidelines do not advocate for evidence-based teaching approaches such as active learning principals (quizzing, spaced learning, and flipped classroom) [9-11] potentially exacerbating variability and insufficiency. 
Taken together, publications documenting deficiencies in radiation oncology topic coverage and the paucity of literature on evidence-based teaching techniques in radiation oncology argue for an understanding of current approaches. Therefore, the goal of this study was to conduct a survey of radiation oncology program directors to characterize radiation oncology resident didactics, as an entryway to identifying knowledge gaps and areas for improvement.

\section{Methods and Materials}

First, we obtained IRB review of this project with subsequent exemption. We then developed a survey instrument to evaluate the educational landscape of resident didactics nationally. We first identified two themes: how didactics are taught and which topics that are covered. We also identified 6 domains of interest, namely, program characteristics, teaching faculty, formal teaching time, instructional approaches for formal teaching, curricular topics, and satisfaction with resident didactics, which we used to create a survey blueprint. We then drafted questions in an iterative, collaborative fashion, which were reviewed for clarity and content by seven local experts in medical education, each with experience in educational measurement and leadership. We included a free text response question regarding ideas to improve resident didactics. We then piloted the instrument with nine radiation oncology physicians not part of the respondent group and conducted cognitive interviews with these participants [12]. The final version of the instrument incorporated feedback from all these sources and is included in Appendix A.

We subsequently created an online version of the survey in Qualtrics (Provo, UT). We distributed a link to the survey in May 2019 to all 91 radiation oncology program directors in the USA, via the Association for Directors of Radiation Oncology Programs (ADROP) email survey list. The survey contained information about the voluntary and anonymous nature of the survey and included consent language signaling agreement to participate in the study if the survey was completed. We sent a reminder email a week later. Multiple responses from the same IP address were excluded. No incentives were offered to solicit responses.

We tabulated the results by question and domain. We performed simple content analysis of free response answers, conducted by review by the corresponding author.

\section{Results}

We received 24 responses out of 91 recipients (26\% response rate). The average number of residents in each program was 10 with a standard deviation of 5 , and the average number of faculty in each program was 16 with a standard deviation of 12. The average hours devoted to resident didactics per week was six (minimum 4, maximum 12). In addition to residents, learners included medical students $(n=21,88 \%)$ and fellows $(n=3,7 \%)$. A minority of additional learners included veterinary radiation oncology residents, radiation therapy students, physicists, and dosimetrists.

The teaching faculty most commonly responsible for didactics were residents and faculty within the program; no fellows participated in teaching in any program. Other teachers included medical students and visiting faculty. The average time a faculty member was present during didactics was $79 \%$ [20-100\%]. The most common role of the faculty member was lecturer ( $96 \%$ of the time); however, other roles included facilitator or observer. All but one program director $(96 \%)$ stated that resident didactic time was protected from clinical responsibilities, but 8/24 (33\%) reported interruptions "sometimes" or "often" due to clinical responsibilities.

In terms of evidence-based teaching practices, while all programs used lecture-based teaching, the majority used quizzing (67\%), and only a minority used simulation (38\%) and flipped classroom (17\%). Other than PowerPoint, the most common electronic resource was quizzing/polling, webinar, and econtour.org (used in 2 programs).

The five most common topics included in didactics were radiation physics/dosimetry, treatment planning, surgical oncology, medical oncology, and palliative care. These topics were included in at least $87 \%$ of the programs. The five least common topics included in didactics were financial success, public speaking/presentation skills, leadership, grant writing, and billing. These topics were included in less than a third of the programs.

Overall, three-quarters of program directors were moderately satisfied or extremely satisfied with resident didactics. However, there were two program directors who were slightly or moderately dissatisfied with the current state of their resident didactics. From the free text comments, we identified some areas for improvement. Program directors cited the desire for increased faculty involvement and sharing of resources across residency programs. Other topics that program directors wished to see included in resident didactics were ethics, finance, practice participation, quality, contouring, simulations, grant writing, interviewing, and research/educational scholarship. One comment from a program director was "for a small program, it's challenging to have consistent quality for didactics. I would [like to] have our program participate in multi-program live webinars and audience participation more often."

\section{Discussion}

We conducted a national survey of program directors in radiation oncology to identify the content and delivery of resident didactics. We defined the current state of resident 
didactic education and found that there was a lack of consistent use of evidence-based teaching practices, a need for incorporation of contemporary topics, and multiple ideas for improvement.

First, we found that the programs contained a diversity of learners including veterinary students, radiation therapy students, physicists, and dosimetrists. A diversity of learners could help to facilitate the discussion of complex topics with ideas from students of different backgrounds. In regard to the teaching faculty, we were surprised to see that no fellows participated in the teaching at any program. As fellowship programs are relatively rare, this may be because few of the responding programs contained fellowships. Most program directors said that resident didactic time was protected, but $1 / 3$ of respondents reported interruptions due to clinical responsibilities. Because program directors were answering this survey (instead of residents), it is possible that this may actually be an underrepresentation of the interruptions. Interruptions in learning could lead to impaired understanding and retention.

Evidence-based teaching practices such as simulation and flipped classroom made up a minority of the educational tools used for radiation oncology didactics. In the flipped classroom model, students obtain baseline knowledge at home and, in class, work through concepts/problem-solving with their peers under the mentorship of their teacher. This technique has been used in other disciplines with some success and is gaining popularity [13]. It is possible these techniques make up a minority of educational tools in radiation oncology didactics because it can be labor-intensive to reconstruct a curriculum that has been present for many years. It also requires faculty to be present and engaged during didactics, increasing the duties and responsibilities of the faculty members.

Despite general satisfaction with resident didactics, we found common themes for improvement which include diversity in didactic topics, increased faculty involvement, and sharing of resources across programs. Even though the program directors voiced a desire to include certain didactic topics, it is unclear why they have not already been included. Barriers to implementation of new didactic topics could be time, money, or a feeling of adequacy with the current curriculum. Similar issues could surround the need for increased faculty development. Future research on barriers to implementation could lead to opportunities for improvement.

Sharing of resources across programs may provide a solution to the depth and breadth of topics needed in resident education and diversity of faculty and instruction. Until recently, there has been a paucity of data outlining the sharing of resources across programs. A reluctance to share resources could be a manifestation of a feeling of competition rather than collaboration amongst residency programs.
Advances in technology such as seamless video conferencing and webinars have lowered technological barriers previously limiting its implementation. An electronic sharing resource can be used as a way to electronically "share" didactics across programs for the common interest of training successful radiation oncologists across the country. Furthermore, sharing of radiation oncology educational resources could help to ensure smaller programs provide high-quality didactics, who may be at a disadvantage because of limited resources, faculty, technology, and patients [14].

To that end, there have been many recent contributions to the radiation oncology online learning landscape. Recently, ARRO has launched a plethora of web-based initiatives including "Meet Me in Treatment Planning," "Clinical Radiation Oncology and eContouring;" "Medical Physics, Radiobiology, and Cancer Biology;" "ARRO Annual Seminar," and "Professional Development and Research" [15]. In addition, there is a growing number of national initiatives such as High Yield Physics Video Series (Hi-Phy) Pilot for Radiation Oncology Trainees [16] and many shared resources provided by the Radiation Oncology Education Collaborative Study Group (ROECSG) including Introduction to Radiation Oncology (IROC), Statistics, and multiple links to other web resources [17]. As part of the response to the COVID-19 pandemic, the segregation and isolation of residents and programs may have fueled, in part, interest in the sharing of electronic resources nationally.

Despite this recent push to share resources, there continues to be a lack of detailed national core curriculum for residents in radiation oncology. In contrast to radiation oncology, in the field of medical oncology, there is a detailed global core curriculum formed by a unified task force from ESMO and ASCO [18]. It describes the competencies necessary to evaluate and treat patients with cancer as a successful medical oncologist. The field of radiation oncology could benefit from a similar national or global standardized, holistic, forward thinking curriculum, a product of collaboration amongst national stakeholders (ASTRO, ABR, ACGME) [19]. Recently presented at the ROECSG 2021 annual meeting, a committee of experts has embarked on a Delphi consensus project to define the core curriculum for radiation oncology residents [20], and we look forward to their findings.

The biggest limitation of this study, which is common to survey studies, is the low response rate. This low response rate may have selected for programs more invested in the educational process and therefore may have overestimated the prevalence of active learning strategies and diversity of topics, potentially minimizing the severity of the issue. A low response rate may also indicate limited generalizability. We attempted to increase our response rate by sending a reminder email to complete the study; a financial incentive may have been helpful as well. Opportunities to increase the 
response rate with incentives or other types of reminders could be used in future studies.

\section{Conclusion}

The lack of a national, standardized, radiation oncology residency didactic curriculum results in variability in resident training. This study supports the continued re-evaluation and reform of old curricula, teaching techniques, and shared resources.

Supplementary Information The online version contains supplementary material available at https://doi.org/10.1007/s13187-021-02080-5.

Authors' Contribution All authors contributed to this manuscript.

Data Availability Available upon request.

Code Availability Available upon request.

\section{Declarations}

Ethics Approval This project was determined to be exempt by the Beth Israel Deaconess Medical Center institutional review board.

Consent to Participate Participants consented to participate when starting the survey.

Consent for Publication All authors give approval for publication of this manuscript.

Competing Interests a. Matthew Abrams receives consulting fees from Medtronic.

b. Dr. Golden reports funding from the National Institutes of Health, Radiation Oncology Institute, and Bucksbaum Institute for Clinical Excellence. He is a manager of RadOncQuestions LLC.

c. Grace Huang declares no competing interests.

\section{References}

1. Burmeister J, Chen Z, Chetty IJ et al (2016) The American Society For Radiation Oncology's 2015 core physics curriculum for radiation oncology residents. Int J Radiat Oncol Biol Phys 95:12981303. https://doi.org/10.1016/j.ijrobp.2016.03.012

2. Woloschak G (2019) Radiation and cancer biology study guide. https://www.astro.org/ASTRO/media/ASTRO/AffiliateP ages/arro/PDFs/Radiobio_StudyGuide.pdf. Accessed 5/15/2021

3. Holliday EB, Swanick CW, McAvoy SA et al (2016) Development of a comprehensive clinical radiation oncology resident didactic curriculum. J Am Coll Radiol 13:1514-1516.e6. https://doi.org/ 10.1016/j.jacr.2016.06.031

4. Royce TJ, Davenport KT, Dahle JM (2019) A burnout reduction and wellness strategy: personal financial health for the medical trainee and early career radiation oncologist. Pract Radiat Oncol 9:231-238. https://doi.org/10.1016/j.prro.2019.02.015
5. Mouw KW, Beck TF, Keen JC, Dicker AP (2018) Assessing the training and research environment for genomics, bioinformatics, and immunology in radiation oncology. JCO Clin Cancer Inform 1-9. https://doi.org/10.1200/cci.18.00045

6. Wei RL, Colbert LE, Jones J et al (2017) Palliative care and palliative radiation therapy education in radiation oncology: a survey of US radiation oncology program directors. Pract Radiat Oncol 7:234-240. https://doi.org/10.1016/j.prro.2016.11.009

7. Pembroke CA, Alfieri J, Biron A et al (2018) Creation of an educational quality improvement program for radiation oncology residents. Pract Radiat Oncol 8:81-89. https://doi.org/10.1016/j. pro.2017.11.007

8. Cabrera AR, Lee WR, Madden R et al (2011) Incorporating gross anatomy education into radiation oncology residency: a 2-year curriculum with evaluation of resident satisfaction. J Am Coll Radiol 8:335-340. https://doi.org/10.1016/j.jacr.2010.10.005

9. The Royal College of Physicians and Surgeons of Canada (2014) Specialty Training Requirements in Radiation Oncology Version $2.0 \mathrm{https}: / / \mathrm{www}$.google.com/searchclient=safari\&rls=en\&q= royal+college+specialty+training+requirements+in+radiation+ oncology \&ie=UTF-8\&oe=UTF-8. Accessed 12/1/2020

10. The Royal Australian And New Zealand College of Radiologists (2012) Radiation Oncology Training Program Curriculum. https:// www.ranzcr.com/college/document-library/radiation-oncologytrainingprogram-curriculum. Accessed 12/1/2020

11. Benstead K, Lara PC, Andreopoulos D, et al (2019) Recommended ESTRO core curriculum for radiation oncology/radiotherapy 4th edition. Radiother Oncol 141:1-4. https://doi.org/10. 1016/j.radonc.2019.08.013

12. Willis GB, Artino AR (2013) What do our respondents think we're asking? Using cognitive interviewing to improve medical education surveys. J Grad Med Educ 5:353-356. https://doi.org/10. 4300/jgme-d-13-00154.1

13. Bishop JL, Verleger MA (2013) The flipped classroom: a survey of the research. In: ASEE Annual Conference and Exposition, Conference Proceedings

14. Emanuel EJ (2020) The inevitable reimagining of medical education. JAMA - J Am Med Assoc 19104:E1-E2. https://doi.org/10. 1001/jama.2020.1227

15. ARRO education resources - webinars. https://www.astro.org/Affil iate/ARRO/Resident-Resources/Educational-Resources/Webinars. Accessed 7 Jun 2021

16. Peters GW, Lincoln H, Tang X et al (2020) High yield physics video series (Hi-Phy) pilot for radiation oncology trainees. Int $\mathrm{J}$ Radiat Oncol 108:e444. https://doi.org/10.1016/j.jirobp.2020.07. 2540

17. ROECSG. https://roecsg.org. Accessed 7 Jun 2021

18. Hansen HH, Bajorin DF, Muss HB et al (2004) Recommendations for a global core curriculum in medical oncology. Ann Oncol 15:1603-1612. https://doi.org/10.1093/annonc/mdh447

19. Golden DW (2020) United States radiation oncology curriculum development: the tail is wagging the dog. Int J Radiat Oncol Biol Phys 106:e1-e4. https://doi.org/10.1016/j.ijrobp.2019.11.399

20. ROECSG core curriculum. https://roecsg.org/corecurriculum/. Accessed 7 Jun 2021

Publisher's Note Springer Nature remains neutral with regard to jurisdictional claims in published maps and institutional affiliations. 